\title{
OCIMUM AMERICANUM L. ESSENTIAL OIL EXHIBITS ANTIMICROBIAL ACTIVITY AGAINST ORAL BACTERIA RELATED TO PERIODONTAL DISEASE
}

\section{(C) Thaweboon Sroisiri, Thaweboon Boonyanit}

\author{
Faculty of Dentistry, Mahidol University, Bangkok, Thailand
}

Ocimum americanum $\mathrm{L}$. or hoary basil is an annual herbaceous plant native to Asia and Africa which has attracted increased interest due to its antimicrobial activity against a wide range of pathogenic microorganisms. To evaluate the antimicrobial property of essential oil extracted from the leaves of Ocimum americanum against oral bacteria related to periodontal disease. Three species of periodontal pathogens including Porphyromonas gingivalis W50, Prevotella intermedia ATCC 25611 and Fusobacterium nucleatum ATCC 25586 were included in the study. Agar diffusion was performed initially to screen the antimicrobial activity of Ocimum americanum essential oil. Minimum inhibitory concentration (MIC) and Minimum bactericidal

concentration $(\mathrm{MBC})$ were then determined using the Millipore membrane method. In the agar diffusion, essential oil extracted from Ocimum americanum exhibited antimicrobial activity against all test bacteria with the zone of inhibition ranging from 24 to $30 \mathrm{~mm}$. The MIC values against $P$. gingivalis and $P$. intermedia were $0.35 \mathrm{mg} / \mathrm{mL}$ whereas that of $F$. nucleatum was $0.70 \mathrm{mg} / \mathrm{mL}$. The $\mathrm{MBC}$ values against $P$. gingivalis and $P$. intermedia were $0.70 \mathrm{mg} / \mathrm{mL}$ whereas that of $F$. nucleatum was $1.4 \mathrm{mg} / \mathrm{mL}$. Ocimum americanum essential oil has an antimicrobial activity which may be a beneficial component of oral health care products to control or prevent periodontal disease by reducing these bacteria in the oral cavity.

\section{BREEDING AND SELECTION OF HIGH QUALITY PLANTS OF RHODIOLA ROSEA}

\section{(C) Thomsen Mette Goul}

Norwegian Institute for Agricultural and Environmental Research (Bioforsk), Kapp, Norway

The composition and content of chemical compounds are one of the most important factors when breeding medicinal plants. The chemical composition in $R$. rosea is mainly influenced by genetic factors but also factors like temperature and day length strongly affects the content of compounds. Furthermore, clonal differences may exist in response to growth conditions and affect the concentration of metabolites. Five Norwegian clones of $R$. rosea, two male and three female clones, from a clone collection was selected on the basis of the content of the marker compounds, total Rosavins and Salidrosid. The seeds from each of the female clones were collected and seed propagated plants from these three were planted at six different locations in Norway. After four seasons the plants were analysed for content of secondary metabolites.

\begin{tabular}{|c|c|c|c|c|c|c|c|}
\hline \multirow[b]{3}{*}{ Clone } & \multicolumn{6}{|c|}{ Content of Tot. Rosavines ( \% of Dry matter) } & \multirow[b]{3}{*}{ Mean } \\
\hline & \multicolumn{6}{|c|}{ Location } & \\
\hline & $64^{\circ} 11^{\circ}$ & $63^{\circ} 08^{\circ}$ & $61^{\circ} 5^{\circ}$ & $60^{\circ} 10^{\circ}$ & $60^{\circ} 11^{\circ}$ & $60^{\circ} 10^{\circ}$ & \\
\hline 1 & 6,831 & 3,730 & 4,81 & 4,171 & 5,127 & 4,251 & 4,82 \\
\hline 2 & 7,064 & 4,626 & 4,331 & 4,757 & 6,179 & 4,683 & 5,27 \\
\hline 3 & 7,381 & 2,974 & 3,373 & 4,069 & 3,799 & 3,916 & 4,25 \\
\hline Mean & 7,09 & 3,78 & 4,17 & 4,33 & 5,04 & 4,28 & \\
\hline
\end{tabular}

The results indicate that the content of secondary metabolites varies with location and also the individual clone's response to location varies (table below). Germplasm collections with accessions that are selected based on their chemical composition are therefore highly valuable. They may carry genes that can be used to improve the current $R$. rosea clones and introduction of new germplasm could further enhance the successful development of improved $R$. rosea genotypes. Hence, crossing between clones with identified content of valuable compounds is one way of achieving high quality genotypes. 